Pesq. Vet. Bras. 35(6):525-530, junho 2015

DOI: $10.1590 / \mathrm{S} 0100-736 \mathrm{X} 2015000600006$

\title{
Método FAMACHA para detectar anemia clínica causada por Haemonchus contortus em cordeiros lactentes e ovelhas em lactação ${ }^{1}$
}

\author{
Maria Angela M. Fernandes ${ }^{2 *}$, Susana Gilaverte ${ }^{2}$, Andréia Buzatti ${ }^{2}$, Lew K. Sprenger ${ }^{3}$, \\ Cláudio J.A. Silva², Mylena T.P. Peres², Marcelo B. Molento ${ }^{3}$ e Alda L.G. Monteiro ${ }^{2}$
}

\begin{abstract}
Fernandes M.A.M., Gilaverte S., Buzatti A., Sprenger L.K., Silva C.J.A., Peres M.T.P., Molento M.B. \& Monteiro A.L.G. 2015. [FAMACHA method for detecting clinical anemia caused by Haemonchus contortus in suckling lambs and lactating ewes.] Método FAMACHA para detectar anemia clínica causada por Haemonchus contortus em cordeiros lactentes e ovelhas em lactação. Pesquisa Veterinária Brasileira 35(6):525-530. Laboratório de Produção e Pesquisa em Ovinos e Caprinos, Departamento de Zootecnia, Universidade Federal do Paraná, Rua dos Funcionários 1540, Curitiba, PR 80035-050, Brazil. E-mail: angela.ufpr@gmail.com

The control of gastrointestinal parasites in small ruminants has traditionally involved the indiscriminate use of anthelmintic drugs that resulted in a high parasite selection pressure for anthelmintic resistance. Selective methods (ex.: FAMACHA/FMC and faecal egg count/EPG), which have good sensitivity to identify animals that need drug treatment, are important alternative methods for parasite control. The objective of this study was to determine the sensitivity and specificity of the FMC method to detect clinical anemia caused by Haemonchus contortus in two highly susceptible categories of Suffolk sheep; suckling lambs and lactating ewes. The ocular conjunctivae of 42 suckling lambs and 35 lactating ewes were evaluated using the FMC method at 14 days interval for five months. Hematocrit (Ht) served as the gold standard for anemia evaluation. To calculate the sensitivity and specificity of the FMC method, different criteria were adopted: animals classified as 4 and 5 , or 3, 4 and 5, were considered to be anemic (positive test), and animals classified as 1, 2 and 3 , or 1 and 2 were considered to be non-anemic (negative test). Three standard values of $\mathrm{Ht}(\leq 22 \%, \leq 19 \%$ or $\leq 15 \%)$ were used to confirm anemia. Among the prevalent genera of helminths $H$. contortus (61.5\%) and Ostertagia sp. (21.3\%) were the most predominant species in the coproculture. For both categories, the inclusion of FMC 3 as a positive test increased the sensitivity but decreased the specificity of the method. When FMC 3 was included as anemic, the percentage of false negative was close to zero, but a greater number of treatments were applied to non-anemic animals (false positive). Most of the lambs (73\%) and ewes (57\%) remained in categories 1 or 2 throughout the trial. Only $5 \%$ of the lambs and $8 \%$ of the ewes were classified as the two major stages of anemia ( 4 and 5 ). The results indicate that the FMC is a safe method to be used in lambs and lactating ewes with clinical anemia from $H$. contortus infection. For both categories, it was necessary to include FMC 3 as a positive condition to increase the sensitivity of the method and to reduce the risk of death of undiagnosed anemic animals (false negatives).
\end{abstract}

INDEX TERMS: FAMACHA method, anemia, Haemonchus contortus, lambs, ewes, hematocrit, selective treatment, sensitivity, sheep, specificity.

\footnotetext{
${ }^{1}$ Recebido em 4 de maio de 2015.

Aceito para publicação em 12 de junho de 2015.

${ }^{2}$ Laboratório de Produção e Pesquisa em Ovinos e Caprinos (LAPOC),

Departamento de Zootecnia, Universidade Federal do Paraná (UFPR), Rua
}

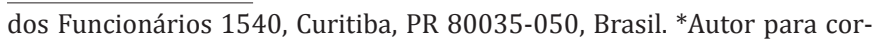
respondência: angela.ufpr@gmail.com

${ }^{3}$ Laboratório de Doenças Parasitárias, Departamento de Medicina Veterinária, UFPR, Rua dos Funcionários 1540, Curitiba, PR 80035-050. 
RESUMO.- 0 controle das endoparasitoses gastrintestinais em pequenos ruminantes tem sido tradicionalmente realizado por meio do uso indiscriminado dos anti-helmínticos, porém essa prática tem resultado em grande pressão de seleção de parasitas resistentes. Métodos seletivos (ex: FAMACHA/FMC e contagem de ovos nas fezes/OPG) e que apresentem boa sensibilidade para identificar os animais que necessitam receber o tratamento químico são importantes alternativas aos métodos tradicionais. 0 objetivo desse trabalho foi determinar a sensibilidade e a especificidade do método FMC, usado para detectar anemia causada por Haemonchus contortus em ovinos da raça Suffolk, em duas categorias de alta suscetibilidade; cordeiros lactentes e ovelhas durante a fase de lactação. A conjuntiva ocular de 42 cordeiros lactentes e 35 ovelhas em lactação foi avaliada usando o método FMC, em intervalos de 14 dias, durante cinco meses. $\mathrm{O}$ hematócrito (Ht) foi utilizado como padrão- ouro para avaliação clínica de anemia dos animais. Para o cálculo de sensibilidade e especificidade, diferentes critérios foram utilizados: animais classificados como 4 e 5 ou 3 , 4 e 5 e anêmicos pelo Ht (teste positivo); animais classificados como 1, 2 e 3 ou 1 e 2 e não anêmicos pelo Ht (teste negativo). Três valores de corte para $\mathrm{Ht}(\leq 22 \%, \leq 19 \%$ ou $\leq 15 \%$ ) foram utilizados para confirmar a anemia. Entre os gêneros observados nas coproculturas, $H$. contortus $(61,5 \%)$ e Ostertagia sp. $(21,3 \%)$ foram os mais prevalentes. Em ambas as categorias, a inclusão do FMC 3 como teste positivo, elevou a sensibilidade mas reduziu a especificidade do método. Quando o FMC 3 foi considerado anêmico, o percentual de falsos negativos foi próximo a zero, no entanto, houve aumento no número de tratamentos aplicados em animais não anêmicos (falso positivo). Durante as avaliações, a maior parte dos cordeiros (73\%) e das ovelhas (53\%) permaneceu nas categorias de FMC 1 e 2. Apenas $5 \%$ dos cordeiros e $8 \%$ das ovelhas foram classificados com os dois maiores graus de anemia (FMC 4 e 5). Os resultados indicam que o FMC pode ser utilizado para identificar os cordeiros lactentes e ovelhas em fase de lactação com sinais clínicos de anemia decorrentes da infecção por $H$. contortus. Para ambas as categorias, foi necessário incluir o FMC 3 como positivo para aumentar a sensibilidade do método; reduzindo o risco de morte de animais anêmicos não diagnosticados (falsos negativos).

TERMOS DE INDEXAÇÃO: FAMACHA, anemia, Haemonchus contortus, cordeiros, ovelhas, especificidade, hematócrito, ovinos, sensibilidade, tratamento seletivo.

\section{INTRODUÇÃO}

A produção e pesquisas com ovinos e caprinos tem crescido consideravelmente em todo o mundo. Os reduzidos índices de produtividade nos rebanhos ovinos são frequentemente associados às infecções por endoparasitas no Brasil, principalmente por Haemonchus contortus. Este parasita apresenta alta patogenicidade e elevada prevalência (Molento et al. 2004, Burke et al. 2007) sendo que as infecções agudas podem causar anemia severa, hipoproteinemia e morte em casos severos (Kaplan et al. 2004, Costa et al. 2007).

As estratégias para controlar os nematoides gastrintestinais têm, tradicionalmente, envolvido o uso de anti- -helmínticos em intervalos fixos e curtos (a cada 30 ou 45 dias), ou no pico de transmissão das larvas, no verão (González-Garduño et al. 2014). Muitas vezes, esse esquema de tratamento preventivo é feito com pouco conhecimento epidemiológico do parasita. Como consequência, tem sido observada alta pressão de seleção de populações de parasitas resistentes com alto índice de homozigose (RR) e perda total de heterogenia para indivíduos suscetíveis (SS) (Fortes \& Molento 2013). Em algumas propriedades brasileiras, a resistência atingiu níveis insustentáveis e conforme relatado por Veríssimo et al. (2012) praticamente nenhum princípio ativo foi capaz de controlar os endoparasitas no estado de São Paulo.

A resistência anti-helmíntica múltipla de $H$. contortus foi destacada como problema na exploração de pequenos ruminantes, em diferentes regiões do mundo (Bartley et al. 2004, Waghorn et al. 2006, McKenna 2010, Veríssimo et al. 2012). Essas observações têm estimulado pesquisadores a buscar alternativas de controle parasitário com o objetivo de reduzir o uso de produtos químicos e a concentração das drogas no meio ambiente, no leite e na carne (Molento et al. 2004, Dobson et al. 2011).

Desde que o método FAMACHA (FMC) foi desenvolvido para o controle seletivo de $H$. contortus em ovinos, sua eficácia foi testada e validada por vários pesquisadores em diferentes países (Van Wyk \& Bath 2002, Kaplan et al. 2004, Molento et al. 2004, Depner et al. 2007, Cringoli et al. 2009, Di Loria et al. 2009, Sotomaior et al. 2012). A adoção do método na seleção de animais que necessitam receber tratamento tem mostrado redução no número de doses e no custo com medicação antiparasitária (Bath \& Van Wyk 2001, Molento et al. 2004). No entanto, Kaplan et al. (2004) destacam que esse método deve ser usado com precaução em cordeiros, pois os animais jovens possuem pequeno volume de sangue, comparados aos ovinos adultos e podem, em um curto período de tempo, progredir de anemia moderada à grave. Essa precaução também deve ser estendida para as ovelhas em lactação devido a redução temporária na imunidade contra os nematoides gastrintestinal (Rahman \& Collins 1992, Kaplan et al. 2004, Miller et al. 2006, Molento et al. 2009).

Dentro desse contexto, o presente trabalho teve por objetivo avaliar a sensibilidade e a especificidade do método FMC para detectar anemia decorrente da infecção por $H$. contortus em ovinos em duas fases do ciclo produtivo, em que são altamente suscetíveis às endoparasitoses, cordeiros lactentes e ovelhas em fase de lactação.

\section{MATERIAL E MÉTODOS}

O estudo foi desenvolvido no Laboratório de Produção e Pesquisa em Ovinos e Caprinos, LAPOC, da Fazenda da Universidade Federal do Paraná, UFPR, localizado em Pinhais, região metropolitana de Curitiba, Paraná, Brasil, entre Janeiro a Maio de 2012. 0 clima é do tipo $\mathrm{Cfb}$, classificação de Köppen, que corresponde ao clima subtropical úmido (mesotérmico).

Todas as avaliações e manejos envolvendo os animais foram realizados de acordo com as normas éticas e aprovados pela Comissão de Ética no Uso de Animais do Setor de Ciências Agrárias da Universidade Federal do Paraná (Protocolo no. 055/2011). 
Durante o período experimental, os cordeiros foram mantidos com suas mães em pastagem de Tifton 85 (Cynodon spp.). Para avaliação do método FMC foram utilizados ovinos em duas fases do ciclo produtivo: 42 cordeiros lactentes e 35 ovelhas na fase de lactação. 0 primeiro grupo foi composto por 20 machos não castrados e 22 fêmeas, provenientes de partos simples ou duplo da raça Suffolk. As avaliações iniciaram quando os cordeiros apresentavam média de 19,7 kg e 42 dias de idade e suas mães 59,6 $\mathrm{kg}$ e 3,5 anos.

A partir do início do experimento (dia zero) e em intervalos de 14 dias, foi realizada a coleta de fezes diretamente da ampola retal de todos os animais. Em cada avaliação, as fezes de todos os animais constituíram um pool de fezes para realização da coprocultura de larvas (Roberts \& O’Sullivan 1950). A identificação das larvas infectantes (L3) foi realizada de acordo com Van Wyk \& Mayhew (2013).

Para ambas as categorias, a avaliação do método FMC foi realizada sempre pelos mesmos três avaliadores que foram treinados de acordo com Van Wyk \& Bath (2002) para comparar a coloração da conjuntiva com o cartão (Bath et al. 2001).

0 hematócrito (Ht) é considerado o padrão-ouro para a avaliação clínica no FMC. Em cada avaliação, o sangue foi coletado por venopunção jugular de todos os animais em tubos com anticoagulante (10\% de EDTA). Os valores de Ht foram determinados pela técnica do micro-hematócrito, utilizando tubos capilares e centrifugação a 10.000 rpm por 5 minutos. Os graus de FMC segundo os valores de Ht são: (1) 28\% e valores acima; (2) 23 a 27\%; (3) 18 a 22\%; (4) 13 a 17\% e (5) 12\% e abaixo (Bath et al. 2001).

Para o cálculo da sensibilidade e especificidade do FMC foram adotados dois critérios: (I) animais com FMC 4 e 5, classificados como anêmicos (teste positivo) e com FMC 1, 2 e 3, não classificados como anêmicos (teste negativo); (II) animais com o FMC 3, 4 e 5, classificados como anêmicos (teste positivo) e FMC 1 e 2, classificados como não anêmicos (teste negativo). Na avaliação da habilidade do método FMC em identificar corretamente os animais que realmente necessitam de tratamento, o valor de corte do hematócrito utilizado para determinar a anemia pode ter grande impacto nos resultados. Devido à falta de valores de referência de Ht para ovinos que sejam diretamente associados com sinais clínicos de anemia (Kaplan et al. 2004, Burke et al. 2007), alguns autores recomendam utilizar mais de um valor de corte para avaliar a sensibilidade do método (Kaplan et al. 2004, Vatta et al. 2001). No presente estudo, foram utilizados três valores de corte: $\mathrm{Ht} \leq 22 ; \mathrm{Ht}$ $\leq 18$ e Ht $\leq 15 \%$, de acordo com Reynecke et al. (2011).

Um resultado verdadeiro positivo foi definido como um animal anêmico (Ht $\leq 22$, $\leq 18$ ou $\leq 15 \%$ ) com pontuação de mucosa ocular pálida (3, 4 e 5 ou 4 e 5). Um resultado falso positivo foi definido como animal que não estava anêmico (Ht $>22$, $>18$ ou $>15 \%$ ) com pontuação de mucosa ocular clara (3, 4 e 5 ou 4 e 5). Um resultado falso negativo foi definido como um animal que estava anêmico com pontuação de mucosa ocular vermelha ou rosa (1 e 2 ou 1, 2 e 3). 0 resultado verdadeiro negativo foi considerado como um animal que não estava anêmico apresentando a conjuntiva vermelha ou rosa. A sensibilidade, especificidade, o valor preditivo do resultado negativo e o valor preditivo do resultado positivo foram calculados de acordo com Vatta et al. (2001), Thrusfield (2005) e Sotomaior et al. (2012). A análise dos dados obtidos incluiu estatística descritiva (média e frequências) e análise de correlação (coeficiente de Pearson). Para avaliar a relação entre os escores de FMC e os valores de $\mathrm{Ht}$, os dados foram distribuídos em gráficos do tipo Box Plot, associados ao coeficiente de Pearson, para cada categoria estudada. As correlações foram calculadas com auxílio do programa estatístico SAS (2002). Os gráficos foram construídos com auxílio do pacote estatístico ACTION versão 2.5 (ESTATCAMP 2013).

\section{RESULTADOS E DISCUSSÃO}

Durante o período de avaliação, os gêneros de helmintos identificados nas coproculturas dos cordeiros e ovelhas foram: Haemonchus sp., Ostertagia sp., Cooperia sp. e Trichostrongylus sp. (Quadro 1). Entre os gêneros observados, Haemonchus sp. $(61,5 \%)$ e Ostertagia sp. $(21,3 \%)$ foram os mais prevalentes durante todo o período. Estes resultados estão de acordo com estudos realizados no Brasil na última década (Molento et al. 2004, Rosalinski-Moraes et al. 2012, Veríssimo et al. 2012). 0 monitoramento por coproculturas a cada 6-8 semanas, a fim de verificar se $H$. contortus é o principal parasito (Bath et al. 2001) é fundamental para aplicação do método FMC. A eficácia do método é maior quando a prevalência de $H$. contortus representar pelo menos $60 \%$ das espécies de helmintos pois esse é o principal parasita que causa anemia nos pequenos ruminantes (Chagas et al. 2007, Bath \& Van Wyk 2009).

De acordo com o Quadro 2, o percentual de animais com Ht baixo e com FMC 4 ou 5 (verdadeiro positivo) foi baixo, tanto para os animais jovens $(2,4$ a $4,3 \%)$ quanto para os animais adultos (2,5 a 5,5\%). Quando o FMC 3 foi incluído como positivo, houve aumento do percentual de cordeiros e ovelhas corretamente diagnosticados como positivos. No entanto, esse percentual de animais verdadeiros positivos,

Quadro 1. Percentual (\%) dos gêneros de nematódeos observados nas coproculturas de cordeiros lactantes e ovelhas durante a fase de lactação. Pinhais/PR, Brasil

\begin{tabular}{lcccccc}
\hline \multirow{2}{*}{ Gênero } & \multicolumn{5}{c}{ Mês de Avaliação (2012) } & \multirow{2}{*}{ Média } \\
\cline { 2 - 6 } & Janeiro & Fevereiro & Março & Abril & Maio & \\
\hline Haemonchus sp. & $74,6 \%$ & $66,0 \%$ & $59,6 \%$ & $35,0 \%$ & $72,2 \%$ & $61,5 \%$ \\
Ostertagia sp. & $13,0 \%$ & $19,3 \%$ & $27,0 \%$ & $33,0 \%$ & $15,5 \%$ & $21,5 \%$ \\
Cooperia sp. & $8,7 \%$ & $6,6 \%$ & $5,1 \%$ & $8,0 \%$ & $2,3 \%$ & $6,1 \%$ \\
Trichostrongylus sp. & $3,7 \%$ & $8,1 \%$ & $8,3 \%$ & $24,0 \%$ & $10,0 \%$ & $10,9 \%$
\end{tabular}

Quadro 2. Número e percentual de verdadeiro positivo, verdadeiro negativo, falso positivo, falso negativo e correto tratamento utilizando o método FAMACHA, de acordo com os diferentes critérios de avaliação (grau de FAMACHA e hematócrito), para cordeiros lactentes e ovelhas em lactação

\begin{tabular}{ccccc}
\hline Verdadeiro & Verdadeiro & Falso & Falso & Correto \\
Positivo & Negativo & Positivo & Negativo & Tratamento \\
\hline
\end{tabular}

Cordeiros lactentes

FAMACHA 4 e 5 considerados como teste positivo

$\mathrm{Ht}^{*} \leq 22 \quad 11(4,3 \%) \quad 233(91,7 \%) \quad 2(0,8 \%) \quad 8(3,2 \%) 244(96,1 \%)$ Ht $\leq 18 \quad 10(3,9 \%) \quad 237(93,3 \%) \quad 3(1,2 \%) \quad 4(1,6 \%) 247(97,2 \%)$ Ht $\leq 15 \quad 6(2,4 \%) \quad 241(94,9 \%) \quad 7(2,7 \%) \quad 0(0 \%) \quad 247(97,2 \%)$ FAMACHA 3, 4 e 5 considerados como teste positivo

$\mathrm{Ht}^{*} \leq 2217(6,7 \%) \quad 183(72,0 \%) 52(20,5 \%) 2(0,8 \%) 200(78,7 \%)$ Ht $\leq 18 \quad 14(5,5 \%) \quad 185(72,8 \%) \quad 55(21,7 \%) \quad 0(0 \%) \quad 199(78,3 \%)$ Ht $\leq 15 \quad 6(2,4 \%) \quad 185(72,8 \%) \quad 63(24,8 \%) \quad 0(0 \%) \quad 191(75,2 \%)$

Ovelhas em Lactação

FAMACHA 4 e 5 considerados como teste positivo

Ht $\leq 22 \quad 13(5,5 \%) \quad 197(83,1 \%) \quad 9(3,8 \%) \quad 18(7,6 \%) 210(92,0 \%)$

Ht $\leq 18 \quad 11(4,6 \%) \quad 207(87,3 \%) \quad 11(4,6 \%) \quad 8(3,4 \%) 218(94,9 \%)$

$\mathrm{Ht} \leq 15 \quad 6(2,5 \%) \quad 212(89,4 \%) \quad 16(6,7 \%) \quad 3(1,3 \%) 218(92,0 \%)$

FAMACHA 3, 4 e 5 considerados como teste positivo

Ht $\leq 2225(10,8 \%) 128(55,4 \%) 75(32,5 \%) \quad 3(1,3 \%) 153(66,2 \%)$

Ht $\leq 18 \quad 18(7,6 \%) \quad 131(55,3 \%) \quad 88(37,1 \%) \quad 0(0 \%) \quad 149(62,9 \%)$

Ht $\leq 15 \quad 9(3,8 \%) \quad 131(55,3 \%) 97(40,9 \%) \quad 0(0 \%) \quad 140(59,1 \%)$

$\overline{{ }^{a} \text { Correto tratamento }}=$ verdadeiro positivo e verdadeiro negativo. 
com ou sem a inclusão do FMC 3, é relativamente pequeno quando comparado com o de verdadeiro negativo. 0 percentual de animais verdadeiro negativo apresentou média acima de 90\% quando o escore 3 não foi incluído como anêmico. Esse percentual de animais não tratados com produtos químicos pode ser fundamental para manter a eficácia do anti-helmíntico por períodos mais longos, pois permite que haja a sobrevivência da população de parasitas sensíveis no meio ambiente (refugia) e com isso, permitiria diminuir a pressão de seleção (Kaplan et al. 2004, Molento 2009).

0 percentual de animais anêmicos não identificados pelo método FMC (falso negativo) variou entre 0 a $7,6 \%$, sendo os percentuais mais elevados observados no $\mathrm{Ht}$ mais alto. Ocorre que, quanto menor o hematócrito, menor a concentração de hemácias e consequentemente mais pálida será a mucosa do animal, portanto, maior será a facilidade do observador em diagnosticar um animal anêmico como verdadeiro positivo. Quando o grau 3 foi incluído na categoria como anêmico, o número de cordeiros e ovelhas anêmicas diagnosticados como saudável pelo método (falso negativo) foi próximo a zero, como também observado por Burke et al. (2007). Ressalta-se que o método FMC é uma avaliação subjetiva, o que permite a classificação equivocada de animais (Rosalinski-Moraes et al. 2012). Van Wyk \& Bath (2002) enfatizaram a importância do treinamento prático de avaliadores para diminuir a subjetividade e garantir o sucesso do método FMC, como verificado em treinamentos no estado do Paraná (Maia et al. 2014).

$\mathrm{Na}$ avaliação da sensibilidade e especificidade do método FMC para identificar corretamente os animais que necessitam de tratamento, estabelecer o limite inferior do Ht é de fundamental importância, pois é o ponto que diferencia os animais anêmicos dos saudáveis. No presente experimento, ficou claro que quanto menor o Ht estabelecido como limite para determinar se um animal estava ou não anêmico, maior foi o risco de erro para um falso negativo. Em geral, há falta de valores de referência para parâmetros hematológicos para ovinos, sobretudo quando se referem aos animais mais jovens. Por isso, em muitos outros estudos, e mesmo na rotina de atendimento clínico, persiste o uso de valores de referência relatados para ovinos adultos $(\mathrm{Ht}=27-45$; Jain 1993) para a avaliação de cordeiros. No entanto, vários fatores de variabilidade, tais como: condições climáticas e ambientais, estado nutricional e estado fisiológico, forma de exploração pecuária, raça, sexo e idade, devem ser considerados para uma adequada avaliação dos parâmetros sanguíneos (Madureira et al. 2013, De Souza et al. 2014).

Apesar do baixo percentual de animais anêmicos não diagnosticados pelo método (falsos negativos), esse poderá se tornar impactante na produção quando consideradas as duas categorias no rebanho (cordeiros e ovelhas), as quais estariam em risco de morte devido o não indicação de tratamento. Além disso, quando o percentual de falso negativo é muito elevado, o teste pode ser questionado quanto à sua precisão na seleção dos animais que necessitam de tratamento. A inclusão do grau 3 como diagnóstico positivo resultou em maior número de tratamentos aplicados em animais não anêmicos (falsos positivos). Resultados semelhantes foram obtidos por Scheuerle et al. (2010) e Soto- maior et al. (2012). No entanto, ao considerar que, no método preventivo todos os animais recebem o anti-helmíntico em intervalos regulares independente de necessitar ou não do tratamento, haveria uma redução considerável no número de tratamentos, apesar do percentual de falsos positivos no método FMC. O percentual de cordeiros tratados corretamente pelo método FMC (resultados verdadeiros negativos + verdadeiros positivo) foi de: $96-97 \%$ (FMC 4 e 5 positivos) e 75-78\% (FMC 3, 4 e 5 positivo). Para as ovelhas em lactação, o percentual foi de $92-94 \%$ quando os FMC 4 e 5 foram considerados positivos e $59-66 \%$ quando o escore 3 foi incluso como positivo. Essa redução no percentual correto de tratamento esta relacionada com a redução do percentual de diagnósticos verdadeiro negativo, também observada por Kaplan et al. (2004) e Di Loria et al. (2009).

No Quadro 3 observa-se que com a inclusão do grau 3 houve aumento da sensibilidade, porém também houve redução na especificidade do método, para ambas as categorias (cordeiros e ovelhas). Valores de sensibilidade mais elevados do que o de especificidade são mais importantes para o método FMC (Vatta el al. 2001). Dessa forma, as consequências em não tratar um animal anêmico (com risco de morte) são mais graves do que tratar um animal que não necessita (não anêmico) de tratamento (Vatta et al. 2001). Portanto, tratar cordeiros com grau 3, 4 e 5 resultará em um maior número de tratamentos sendo aplicados aos animais considerados falsos positivos, mas eleva a sensibilidade do método e reduz a chance de mortalidade em animais anêmicos não diagnosticados. Assim, os dados descritos no Quadro 3, estão de acordo com Vatta et al. (2001) e Van Wyk \& Bath (2002) na África do Sul, Burke et al. (2007) nos Estados Unidos e Sotomaior et al. (2012) no Brasil.

0 valor preditivo positivo é a probabilidade de um animal ser anêmico quando o teste é positivo e o contrário para o valor preditivo negativo (Vatta et al. 2001). Os valores preditivo positivo foram menores com a inclusão do

Quadro 3. Valor preditivo positivo (VPP) e negativo (VPN), sensibilidade e especificidade do método FAMACHA para cordeiros lactentes e ovelhas em lactação, de acordo com os diferentes critérios de avaliação (grau de FAMACHA e hematócrito)

\begin{tabular}{llll}
\hline VPP & VPN & Sensibilidade & Especificidade \\
\hline
\end{tabular}

Cordeiros lactentes

FAMACHA 4 e 5 considerados como teste positivo

$\begin{array}{lcccc}\text { Ht } \leq 22 & 84,6 & 96,7 & 57,9 & 99,1 \\ \mathrm{Ht} \leq 18 & 76,9 & 98,3 & 71,4 & 98,7 \\ \mathrm{Ht} \leq 15 & 46,2 & 100 & 100 & 97,2 \\ \text { FAMACHA } & 3,4 \text { e } 5 & \text { considerados } & \text { como teste positivo } & \\ \mathrm{Ht} \leq 22 & 24,6 & 98,9 & 88,89 & 77,9 \\ \mathrm{Ht} \leq 18 & 20,3 & 100 & 100 & 77,1 \\ \mathrm{Ht} \leq 15 & 8,7 & 100 & 100 & 74,6\end{array}$

Ovelhas em lactação

FAMACHA 4 e 5 considerados como teste positivo

Ht $\leq 22 \quad 59,1 \quad 91,6 \quad 41,9 \quad 95,6$

Ht $\leq 18 \quad 50,0 \quad 96,3 \quad 57,9 \quad 94,9$

Ht $\leq 15 \quad 27,3 \quad 98,6 \quad 66,7 \quad 93,0$

FAMACHA 3, 4 e 5 considerados como teste positivo

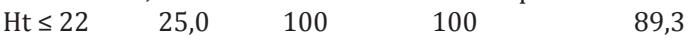

$\begin{array}{lllll}\text { Ht } & 18 & 17,0 & 100 & 100\end{array}$

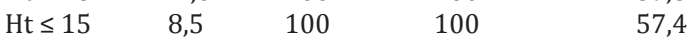


escore 3, tanto para os cordeiros quanto para as ovelhas, devido ao maior percentual de animais falsos positivos. 0 valor preditivo negativo foi alto, acima de $90 \%$, em ambas as categorias, com ou sem a inclusão do FMC 3 como positivo, devido ao elevado percentual de verdadeiro negativo. Kaplan et al. (2004) relataram dados semelhantes após avaliar 847 ovelhas de diferentes idades e raças com o método FMC, provenientes de 39 fazendas nos EUA.

No presente estudo, o Ht e o FMC apresentaram correlação significativa $(\mathrm{P}<0,001)$ e negativa, tanto para os cordeiros $(R=-0,64)$ quanto para as ovelhas $(R=-0,59)$ (Fig.1). Estes resultados permitem concluir que o mé-
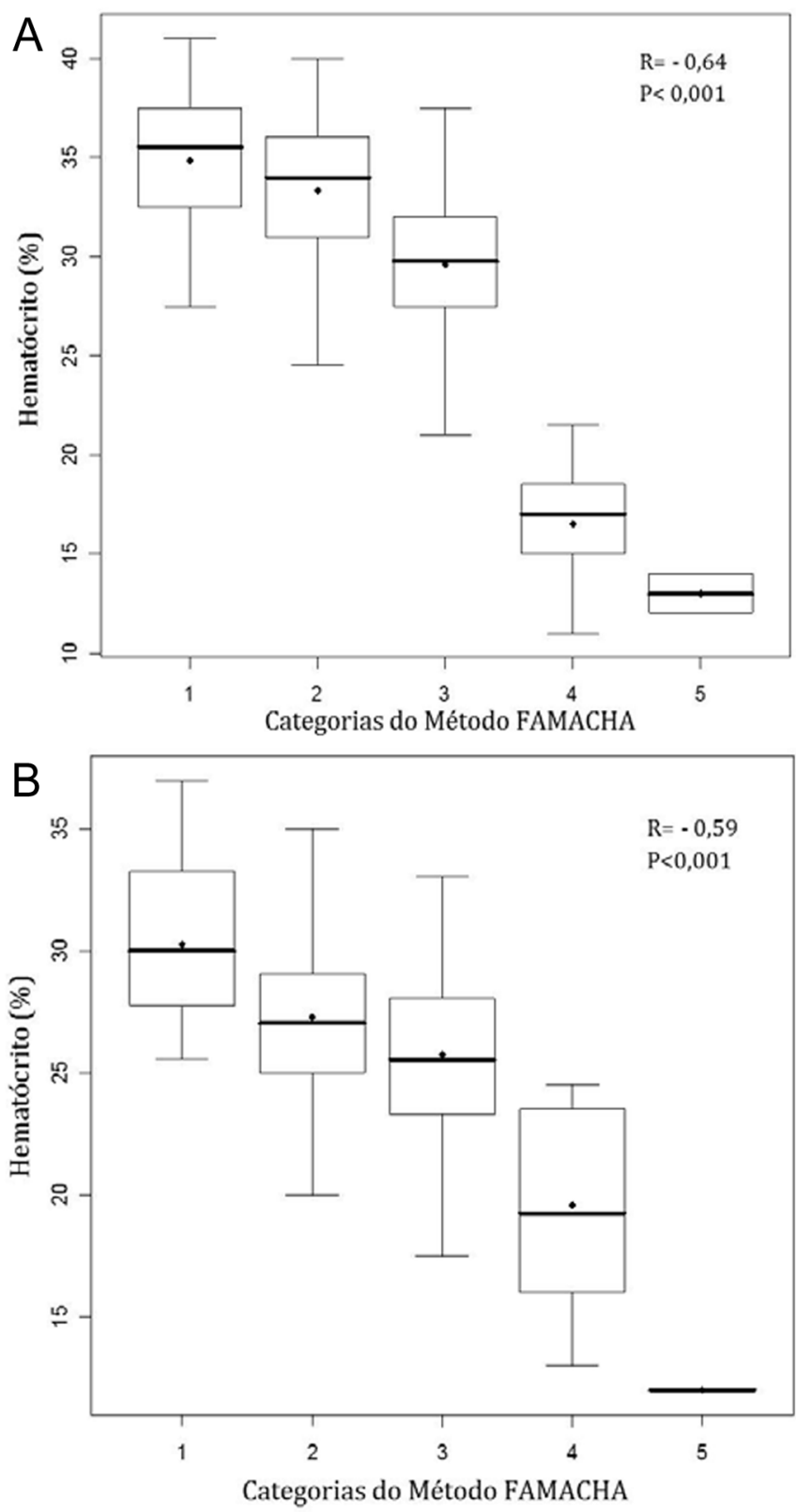

Fig.1. (a) Box plot demonstrando a relação entre o hematócrito e cada categoria do método FAMACHA em cordeiros lactentes e (b) ovelhas em lactação. Bordas inferiores e superiores da caixa representam 25 e 75 percentil, respectivamente. As Correlações de Pearson de cada categoria, cordeiro e ovelha, são exibidas em sua respectiva Figura (a) e (b).
Quadro 4. Médias e desvios-padrão para hematócrito (Ht) de cordeiros lactentes e ovelhas em lactação de acordo com o escore e hematócrito de referência do método FAMACHA

\begin{tabular}{|c|c|c|c|c|c|}
\hline \multirow{2}{*}{\multicolumn{2}{|c|}{$\frac{\text { Escores do FAMACHA }}{\text { Ht do FMC } *}$}} & \multicolumn{2}{|c|}{ Cordeiros Lactentes } & \multicolumn{2}{|c|}{ Ovelhas em Lactação } \\
\hline & & $\mathrm{Ht} \%$ & $\mathrm{~N}=254$ & $\mathrm{Ht} \%$ & $\mathrm{~N}=237$ \\
\hline 1 & $\geq 28$ & $34,5 \pm 3,6$ & $63(24,8 \%)$ & $30,2 \pm 3,2$ & $35(14,8 \%)$ \\
\hline 2 & $23-27$ & $32,5 \pm 4,4$ & $122(48,0 \%)$ & $27,2 \pm 2,9$ & $99(41,8 \%)$ \\
\hline 3 & $18-22$ & $27,9 \pm 6,0$ & $56(22,1 \%)$ & $25,7 \pm 3,8$ & $83(35,0 \%)$ \\
\hline 4 & $13-17$ & $16,5 \pm 7,9$ & $11(4,3 \%)$ & $19,6 \pm 4,2$ & $18(7,6 \%)$ \\
\hline 5 & $\leq 12$ & $13,0 \pm 1,4$ & $2(0,8 \%)$ & $12 \pm 0,0$ & $2(0,8 \%)$ \\
\hline
\end{tabular}

todo foi adequadamente utilizado para ambas as categorias. Os valores de $\mathrm{Ht}$ dos cordeiros foram mais altos para os FMC 1 e 2 (Fig.1a) do que os de Ht das ovelhas (Fig.1b). Nos cordeiros, o valor de Ht mais baixo detectado foi de $11 \%$ e o mais alto de $41 \%$ (Quadro 4). Na categoria ovelhas em lactação, o menor valor de Ht detectado foi de $9 \%$ e o mais alto de $39 \%$. 0 percentual de animais classificados com FMC 1 e 2, durante todo o período experimental, foi de $73 \%$ para os cordeiros e de $57 \%$ para as ovelhas. Apenas $5 \%$ dos cordeiros e $8 \%$ das ovelhas foram classificados com os dois maiores graus de anemia (4 e 5). Esta situação sugere que grande parte dos animais estariam infectados, no entanto, apenas um menor percentual estaria contaminado com a maior parte dos parasitas e provavelmente poderiam necessitar de tratamento (Herbert \& Isham 2000).

Como ambas as categorias são bastante sensíveis às endoparasitoses gastrintestinais, ressalta-se que no presente trabalho os animais foram examinados com grande frequência, o que se considera essencial para o sucesso do método FMC.

\section{CONCLUSÕES}

Foi possível identificar os cordeiros lactentes e as ovelhas em fase de lactação com sinais clínicos de anemia com a utilização do método FMC, decorrentes da infecção por Haemonchus contortus que necessitariam receber tratamento.

Assim, se sugere a inclusão dos animais com FMC grau 3 para receber tratamento, elevando a sensibilidade do método e reduzindo o percentual de animais falso negativos com risco de morte.

\section{REFERÊNCIAS}

Bartley D.J., Jackson F., Jackson E. \& Sargison N. 2004. Characterization of two triple resistant field isolates of Teladorsagia from Scottish lowland sheep farms. Vet. Parasitol. 123:189-199.

Bath G.F. \& Van Wyk J.A. 2001. Using the Famacha system on commercial sheep farms in South Africa. International Sheep Veterinary Congress, Cape Town, South Africa, Vol.1, p.3.

Bath G.F. \& Van Wyk J.A. 2009. The Five Point Check for targeted selective treatment of internal parasites in small ruminants. Small Rumin. Res. 86:6-13.

Burke J.M., Kaplan R.M., Miller J.E., Terril T.H., Getz W.R., Mobini S., Valencia E., Williams M.J., Williamson L.H. \& Vatta A.F. 2007. Accuracy of the FAMACHA system for on-farm use by sheep and goat producers in the southeastern United States. Vet. Parasitol. 147:89-95.

Chagas A.C.S., Carvalho C.O. \& Molento M.B. 2007. Método FAMACHA: um recurso para o controle da verminose em ovinos. Circ. Téc. 52, Embrapa Pecuária Sudeste, São Carlos, SP. 
Costa R.L.D., Bueno M.S., Verissimo C.J., Cunha E.A., Santos L.E., Oliveira S.M., Sposito Filha E. \& Otsuk I.P. 2007. Performance and nematode infection of ewe lambs on intensive rotational grazing with two different cultivars of Panicum maximum. Trop. Anim. Health Prod. 39:255-263.

Cringoli G., Rinaldi L., Veneziano V., Mezzino L., Vercruysse J. \& Jackson F. 2009. Evaluation of targeted selective treatments in sheep in Italy: Effects on faecal worm egg count and milk production in four case studies. Vet. Parasitol. 164:36-43.

Depner R.A. Gavião A.A., Cecim M., Rocha R. \& Molento M.B. 2007. Growth performance in naturally infected lambs with gastrointestinal nematodes under selective treatment with Famacha method and preventive treatment. Arch. Vet. Sci. 12:32-37.

De Souza D.F., Monteiro A.L.G., Dittrich R.L., Schmidt E.M.S., Fernandes S.R. \& Beltrame O.C. 2014. Dinâmica pré e pós-colostral de parâmetros bioquímicos em cordeiros. Ciênc. Anim. Bras. 15:313-321.

Di Loria A., Veneziano V., Piantedosi D., Rinaldi L., Cortese L., Mezzino L., Cringoli G. \& Ciaramella P. 2009. Evaluation of the FAMACHA system for detecting the severity of anaemia in sheep from southern Italy. Vet. Parasitol. 161:53-59.

Dobson R.J., Hosking B.C., Besier R.B., Love S., Larsen J.W.A., Rolfe P.F. \& Bailey J.N. 2011. Minimizing the development of anthelmintic resistance, and optimizing the use of the novel anthelmintic monepantel, for the sustainable control of nematode parasites in Australian sheep grazing systems. Aust. Vet. J. 89:160-166.

ESTATCAMP 2013. Software Action. Disponível em <www.portalaction. com.br> Acesso 5 abr. 2013.

González-Garduño R., Torres-Acosta J.F.J. \& Chay-Canul A.J. 2014. Susceptibility of hair sheep ewes to nematode parasitism during pregnancy and lactation in a selective anthelmintic treatment scheme under tropical conditions. Res. Vet. Sci. 96:487-492.

Fortes F.S. \& Molento M.B. 2013. Resistência anti-helmíntica em nematoides gastrintestinais de pequenos ruminantes: avanços e limitações para seu diagnóstico. Pesq. Vet. Bras. 33:1391-1402.

Herbert J. \& Isham V. 2000. Stochastic host-parasite interaction models. J. Math. Biol. 40:343-371.

Jain N.C. 1993. Essentials of Veterinary Hematology. 4th ed. Lea and Febiger, Philadelphia.

Kaplan R.M., Burke J.M., Terrill T.H., Miller J.E., Getz W.R., Mobini S., Valencia E., Williams M., Williamson L.H., Larsen M. \& Vatta A.F. 2004. Validation of the FAMACHA eye color chart for detecting clinical anemia on sheep and goat farms in the southern United States. Vet. Parasitol. 123:105-120.

Madureira K.M., Gomes V., Barcelos B., Zani B.H., Shecaira C.L., Costa C.R. \& Benesi F.J. 2013. Parâmetros hematológicos e bioquímicos de ovinos da raça Dorper. Semina, Cienc. Agr. 34:811-816.

Maia D., Rosalinski-Moraes F., van Wyk J.A., Weber S. \& Sotomaior C.S. 2014. Assessment of a hands-on method for FAMACHA system training. Vet. Parasitol. 200:165-171.

McKenna P.B. 2010. Update on the prevalence of anthelmintic resistance in gastrointestinal nematodes of sheep in New Zealand. N. Z. Vet. J. 58:172-173.
Miller J.E. \& Horohov D.W. 2006. Immunological aspects of nematode parasite control in sheep. J. Anim. Sci. 84:124-132.

Molento M.B., Tasca C., Gallo A., Ferreira M., Bononi R. \& Stecca E. 2004. Método FAMACHA como parâmetro clínico individual de infecção por Haemonchus contortus em pequenos ruminantes. Ciência Rural 34:1139-1145.

Molento M.B. 2009. Parasite control in the age of drug resistance and changing agricultural practices. Vet. Parasitol. 3:188-196.

Molento M.B., Gavião A.A., Depner R.A. \& Pires C.C. 2009. Frequency of treatment and production performance using the FAMACHA method compared with preventive control in ewes. Vet. Parasitol. 162:314-319.

Rahman W.A. \& Collins G.H. 1992. An association ol faecal egg counts and prolactin concentrations in sera of periparturient Angora goats. Vet. Parasitol. 43:85-89.

Reynecke D.P., Wyk J.A van, Gummow B., Dorny P. \& Boomker J. 2011. Validation of the FAMACHA $@$ eye colour chart using sensitivity/specificity analysis on two South African sheep farms. Vet. Parasitol. 177:203-211.

Roberts F.H.S. \& O' Sullivan J.P. 1950. Methods of egg counts and larval cultures for strongyles infesting the gastrointestinal tract of cattle. Aust. Agric. Res. 1:99-102.

Rosalinski-Moraes F., Fernandes F.G., Munaretto A., De Oliveira S., Wilmsenm O., Pereira M.W. \& Meirelles A.C.F. 2012. Método FAMACHA, escore corporal e de diarreia como indicadores de tratamento anti-helmíntico seletivo de ovelhas em reprodução. Biosci. J. 6:1015-1023.

SAS Institute 2002. SAS Systems for Windows. Version 9th ed. SAS Institute, Inc., Cary, NC.

Scheuerle M., Mahling M., Muntwyler J. \& Pfister K. 2010. The accuracy of the FAMACHA method in detecting anaemia and haemonchosis in goat flocks in Switzerland under field conditions. Vet. Parasitol. 170:71-77.

Sotomaior C.S., Rosalinski-Moraes F., Da Costa A.R.B., Maia D., Monteiro A.L.G. \& Van Wyk J.A. 2012. Sensitivity and specificity of the FAMACHA in Suffolk sheep and crossbred Boer goats. Vet. Parasitol. 190:114-119.

Thrusfield M.V. 2005. Veterinary Epidemiology. 3rd ed. Blackwell, Cambridge, UK. 610p.

Van Wyk J.A. \& Bath G.F. 2002. The FAMACHA system for managing haemonchosis in sheep and goats by clinically identifying individual animals for treatment. Vet. Res. 33:509-529.

Van Wyk J.A. \& Mayhew E. 2013. Morphological identification of parasitic nematode infective larvae of small ruminants and cattle: a practical lab guide, Onderstepoort. J. Vet. Res. 80:14.

Vatta A.F., Letty B.A., Van der Linde M., Van Wijk E.F., Hansen J.W. \& Krecek R.C. 2001. Testing for clinical anaemia caused by Haemonchus spp. in goats farmed under resource-poor conditions in South Africa using an eye colour chart developed for sheep. Vet. Parasitol. 99:1-14.

Veríssimo C.J., Niciura S.C.M., Alberti A.L.L., Rodrigues C.F.C., Barbosa C.F.C., Chiebao D.P., Cardoso D., Silva G.S., Pereira J.R., Margatho L.F.F., Costa R.L.D., Nardon R.F., Ueno T.E.H., Curci V.C.L.M. \& Molento M.B. 2012. Multidrug and multispecies resistance in sheep flocks from São Paulo, Brazil. Vet. Parasitol. 187:209-216.

Waghorn T.S., Leathwick D.M., Rhodes A.P., Lawrence K.E., Jackson R., Pomroy W.E., West D.M. \& Moffat J.R. 2006. Prevalence of anthelmintic resistance on sheep farms in New Zealand. N. Z. Vet. J. 54:271-277. 